\title{
IDENTIFICATION OF THE MATERIAL PROPERTIES OF AN 18650 LI-ION BATTERY FOR IMPROVING THE ELECTROCHEMICAL MODEL USED IN CELL TESTING
}

\author{
BENCE CSOMÓs*1 AND DÉNES FODOR ${ }^{1}$ \\ ${ }^{1}$ Research Institute of Automotive Mechatronics and Automation, University of Pannonia, Egyetem u. 10, \\ Veszprém, 8200, HUNGARY
}

\begin{abstract}
The aim of this paper is to present an application of the generalized Warburg element and Constant Phase Element (CPE) for non-Fickian diffusion modeling. These distributed elements are intended to provide a better fit of low-frequency impedance data than the standard finite-length Warburg element in the case of most batteries. In addition, the current study demonstrates the ambiguity of the finite-length Warburg element if impedance data is insufficient within the verylow-frequency impedance spectrum. In order to select the appropriate Randles circuit for non-Fickian diffusion modeling, several configurations have been investigated. Based on the best fit of impedance data, the State-of-Charge (SoC) dependency of the Randles circuit parameters has also been analyzed. This study concerns a Samsung ICR18650-26F $2600 \mathrm{mAh}$ battery cell which was subjected to Electrochemical Impedance Spectroscopy (EIS) measurements between 10 $\mathrm{mHz}$ and $100 \mathrm{kHz}$ as a function of SoC. The results were plotted and compared in the form of Nyquist plots. The Randles circuit parameters such as the resistances $R_{\mathrm{s}}$ and $R_{\mathrm{ct}}$, double-layer $C_{\mathrm{dl}}$, leaky capacitance CPE and Warburg coefficients were estimated using ZView software. The present paper shows that CPE - and its QPE form - is a recommended choice to yield the best fit in terms of non-Fickian diffusion impedance. In addition, using CPE is a better alternative to avoid problems with initial values and multiple local solutions, which may exist in the case of the Warburg element. The resultant Randles circuit parameters and their SoC characteristics can be effectively used in further electrochemical modeling.
\end{abstract}

Keywords: Li-Ion Battery, Electrochemistry, Material, Battery model, Parameter estimation

\section{Introduction}

The State of Health (SoH) of a battery plays an important role in electric applications since it has a great influence on the available capacity and power of a battery [1]. SoH deteriorates with battery usage and the rate of aging is related to the operating history of the battery. Therefore, it is recommended to track the state variables of a cell throughout its life cycle and adapt the $\mathrm{SoH}$ prediction according to the current condition of the battery cell.

A common and reliable electrochemical model used in Finite Element Analysis (FEA) is based on the work of Newman et al. [2]. It consists of charge and mass balance equations in both solid (electrode) and liquid (electrolyte) materials, which describe the main operating characteristics of the cell. Even though these formulae could describe the behavior of the cell in $3 \mathrm{D}$, due to their high degree of nonlinearity and complexity, Pseudo-2D (P2D) modeling is favorable in terms of FEA [3]. It is also sufficiently representative to model wearout for automotive applications [4]. In Fig. 1, a typical Pseudo-2D structure of a Li-Ion cell can be seen.

\footnotetext{
*Correspondence: csomos.bence@gmail.com
}

Since battery manufacturing is still a developing sector, the electrochemical composition of a cell constantly changes. Therefore, few battery-chemistry standards and complete databases can describe a given cell structure. Insufficiently reliable and valid battery data inhibits battery modeling since a cell must always be inspected to determine its electrochemical parameters before modeling. The standard way to obtain these electrochemical data is usually through an equivalent circuit modeling process with which the electrochemical properties of the cell can be extracted from EIS measurements.

\section{Diffusion modeling techniques}

It is possible to calculate battery-specific data using several techniques, which can basically be grouped into two types in terms of the measurement approach applied:

- direct measurements, which typically require disassembly of the cell, special preparations or an experimental open-cell. These measurements can be, for example, different types of Electron Microscopy (EM), Computed Tomography (CT), titration, postmortem analysis, etc. 


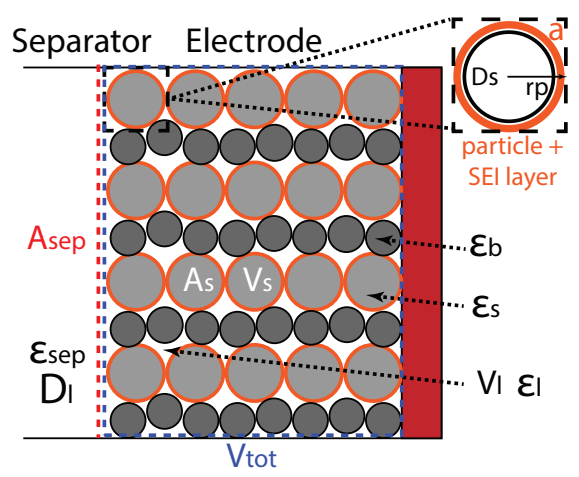

Figure 1: The relationship between the core material parameters and components of the cell. $A_{\text {sep }}$ is addressed to the area of the separator. $\varepsilon_{\mathrm{sep}}, \varepsilon_{\mathrm{b}}, \varepsilon_{\mathrm{s}}$, and $\varepsilon_{1}$ denote the porosities of the separator, binder, solid matrix and void fraction, respectively. $D_{1}$ represents the salt diffusion coefficient in the electrolyte. $D_{\mathrm{s}}$ stands for the diffusion coefficient in the solid electrode. $V_{\mathrm{l}}, V_{\mathrm{s}}$ and $V_{\mathrm{tot}}$ are the volumes of the liquid, solid material and whole electrode, respectively. $r_{\mathrm{p}}$ and a denote the average radius of each electrode particle and the specific surface area of the electrode.

- indirect measurements that do not require disassembly of the cell, e.g. current impulse excitation, Electrochemical Impedance Spectroscopy (EIS), galvanometry, potentiometry, chronoamperometry, etc. [5]

EIS is a well-established and suitable method in the analysis with regard to battery kinetics and has a solid background in the literature [6]. Another advantage of EIS is that it does not require special preparation of the cell that would be extortionate and time-consuming.

Electrochemical parameters are formulated from EIS data in the form of resistive, capacitive, inductive or distributed elements such as the Constant Phase Element (CPE) or Warburg element.

\subsection{Standard equivalent circuits}

In order to obtain battery-specific data, a TransmissionLine Model (TLM) was applied that is introduced and expounded on in [7]. It provides a generalized modeling solution for transport processes in porous electrodes by utilizing a finite number of serially connected resistorcapacitor (RC) pairs in parallel, which resolve the ion and electron transport appropriately. The network obtained in this way is considered to be ambiguous since various arrangements can be reduced to the same circuit resulting in identical resistance and capacitance of the circuit [8]. Although the number of RC pairs used can increase the resolution of the transport process and thus improve its accuracy, the increase in the number of elements results in stability as well as local and global optima problems due to equivocality. Since this is a major disadvantage of extended TLM networks, they are usually avoided.

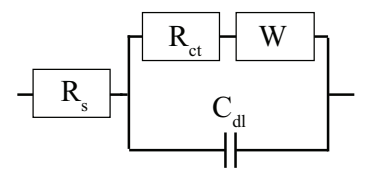

Figure 2: Randles equivalent circuit consisting of serial resistance $R_{\mathrm{s}}$, charge-transfer resistance $R_{\mathrm{ct}}$, double-layer capacity $C_{\mathrm{dl}}$ and distributed impedance element $W$.

A simplified form of TLM is the Randles equivalent circuit model (Fig. 2) which can be obtained if the theoretical RC line of infinite length is reduced to a single Z-distributed element. In other words, the Z-distributed element models the limiting case of TLM. Its fundamental principles and mathematical background are comprehensively described in Barsoukov's book [9]. The standard Randles circuit model couples together the individual characteristics of electrodes and electrolytes into one corresponding circuit element. The Randles circuit model consists of relaxation components such as the serial resistance of the electrolyte $R_{\mathrm{s}}$, charge transfer resistance $R_{\mathrm{ct}}$, a double-layer capacitor $C_{\mathrm{dl}}$ and distributed impedance for modeling the low-frequency diffusive behavior of the cell. Usually, the double-layer effect exhibits non-ideal capacitive behavior so a CPE - the equivalent to a "leaky" capacitor - should be used instead of a $C_{\mathrm{dl}}$. All of these detailed parameters of both electrodes are grouped together, thus they represent the combined behavior of the two electrodes.

Ion transport that occurs in the low-frequency bandwidth of the impedance spectrum can involve diffusion, migration and convection, however, only diffusion is of interest because distributed elements are responsible for diffusion modeling. Diffusion modeling is the bottleneck of model development since it is related to a complex and interleaved electrochemical process that mainly characterizes the long-term operation of the battery. Ion transport can be, by and large, divided into two parts, namely lithium diffusion in the solid active electrode material and electrolyte. The "tail" at the beginning of the lowfrequency bandwidth in the Nyquist plot shows diffusion in the electrolyte while the transition from the tail through an arc to a straightened end of the impedance curve depicts the solid-phase diffusion inside the electrode matrix (Fig. 3). In fact, the cathode is composed of a poor ionconducting material. A general rule of thumb is that its diffusion coefficient is $2-4$ orders of magnitude smaller than the diffusion coefficient of lithium ions in the electrolyte, that is, the salt diffusion coefficient of the electrolyte $D_{1}$ falls within the range of $10^{-10}-10^{-11} \mathrm{~m}^{2} / \mathrm{s}$ while the diffusion coefficient of lithium ions $D_{\mathrm{s}}$ in the solid matrix falls between $10^{-13}$ and $10^{-14} \mathrm{~m}^{2} / \mathrm{s}$. In other words, the time constant for diffusion of Li-Ion transport is smaller in the electrolyte ( $\sim 10-100$ s) than in the solid matrix ( $>100 \mathrm{~s}$ ). Consequently, the two types of diffusion need to be separated and should be modeled based on their different characteristic impedances. 


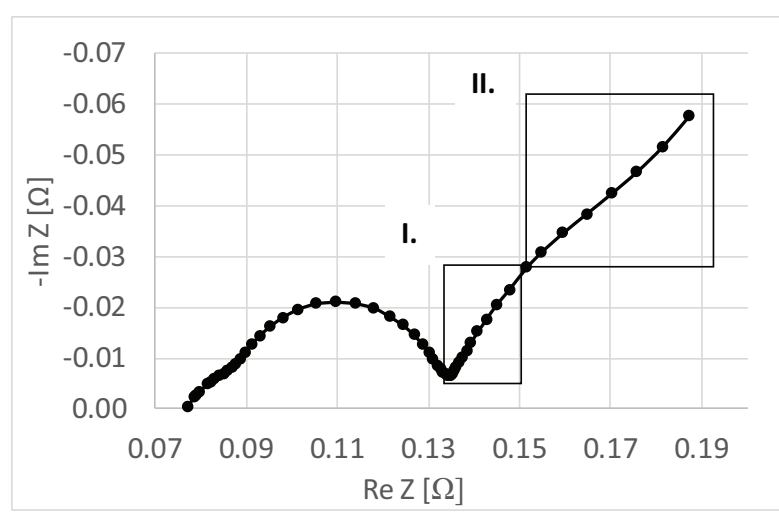

Figure 3: A typical Nyquist plot of a Li-Ion cell; I. denotes a "tail" that mainly represents diffusion in the electrolyte; II. shows a transition from liquid-phase to solid-phase diffusion.

Generally, diffusion is modeled by the classical Warburg impedance in accordance with the following assumptions: diffusion is Fickian (planar diffusion); the electrolyte is supporting, symmetric and binary; the cell remains in a quasi-equilibrium state during excitation; and no reaction occurs in the bulk of the electrolyte. Under these premises, the standard Warburg impedance has an exponent of 0.5 that implies its $45^{\circ}$ phase angle. If diffusion occurs in an infinite reservoir where the concentration can decrease to zero, infinite-length Warburg impedance can be assumed, otherwise diffusion is restricted and finite-length reflective or transmissive Warburg impedance can be assumed depending on whether the equivalent circuit is terminated by an open circuit or a resistor, respectively. The former and latter cases can be mathematically expressed by extending the standard infinite-length Warburg impedance with the hyperbolic functions tanh and coth, respectively. All three types of Warburg elements exhibit the same $45^{\circ}$ gradient at the beginning of the low-frequency bandwidth in the Nyquist plot.

In terms of impedance, using finite-length Warburg elements is unsuitable if an insufficient number of data points in the low-frequency bandwidth of the impedance spectrum are available to fit the hyperbolic functions well. This occurs when the EIS measurements typically run up until $10 \mathrm{mHz}$ but some cells do not show a clear and distinct effect of diffusion in the solid phase. In this case, only the tail part of the impedance spectra can be reasonably modeled. Due to a lack of low-frequency data points, the finite-length Warburg elements cannot be effectively applied. On the other hand, the tail part of the diffusion impedance can be modeled by CPE according to $[11,12]$ which is a similar but more robust alternative to the Warburg elements.

All the transfer functions of the standard types of distributed elements are summarized in Table 1. The transfer function of CPE can be expressed in two different forms according to the position of its time constant for diffusion
Table 1: Transfer functions of standard distributed elements used in Fickian diffusion modeling [10]. $\sigma$ denotes the Warburg coefficient, $\omega$ represents the excitation frequency, $\tau_{\mathrm{D}}$ stands for the diffusion time constant, $Q$ is the QPE time constant, $j$ denotes the imaginary unit and $R_{\mathrm{w}}$ represents the Warburg resistance.

\begin{tabular}{c|c}
\hline Name & Impedance \\
\hline $\begin{array}{c}\text { infinite-length } \\
\text { Warburg } \\
\text { finite-length } \\
\text { reflective Warburg } \\
\begin{array}{c}\text { finite-length } \\
\text { transmissive Warburg }\end{array}\end{array}$ & $Z_{\mathrm{ilw}}(j \omega)=\frac{R_{\mathrm{w}}}{\sqrt{j \omega \tau_{\mathrm{D}}}}=\sigma \frac{1}{\sqrt{\omega}}(1-j)$ \\
CPE & $Z_{\mathrm{fltw}}(\omega)=R_{\mathrm{w}} \operatorname{coth} \frac{\left(\sqrt{j \omega \tau_{\mathrm{D}}}\right)}{\sqrt{j \omega \tau_{\mathrm{D}}}}$ \\
$\mathrm{QPE}$ & $Z_{\mathrm{CPE}}(\omega)=\frac{1}{\sqrt{j \omega \tau_{\mathrm{D}}}}$ \\
& $Z_{\mathrm{QPE}}(\omega)=\frac{1}{Q \sqrt{j \omega}}$ \\
\hline
\end{tabular}

$\tau_{\mathrm{D}}$. If $\tau_{\mathrm{D}}$ is emphasized from its square root, it is referred to as QPE, which sometimes provides a more stable regression than CPE.

Up to this point, only Fickian diffusion has been considered that can be clearly identified by its square-rootlike frequency dependency in terms of the transfer function of the distributed elements. However, the impedance fit becomes more interesting when the cell impedance exhibits non-Fickian behavior, that is, the phase angle is not $45^{\circ}$ due to diffusion non-idealities. These phenomena were investigated and are mostly related to multi-phase and multi-scale diffusion in porous electrodes [13, 14], diffusion coupled with migration $[15,16]$ and/or diffusion in non-conventional space [17-19]. Non-Fickian diffusion can be treated by fractional order circuits which consist of various types of typical configurations. In the literature [20], Warburg impedance is referred to as "generalized" if it reflects the fractional intent. In this case, the Warburg exponent is generalized and denoted by $\gamma$, the dispersion parameter.

\subsection{Equivalent circuit development for model- ing non-Fickian diffusion}

In order to obtain the most accurate fit of the impedance curves, several configurations of the Randles circuit were developed. These setups are presented in Fig. 4. The key differences between them can be explored in terms of both the position and type of the distributed elements in the circuit. In some papers from the literature, the distributed element is in series with $R_{\mathrm{ct}}$ [21], while in others, it is placed in series with the parallel $R_{\mathrm{ct}}-C_{\mathrm{dl}}$ or $R_{\mathrm{ct}}-\mathrm{CPE}$ branch. The distributed element can be either the Warburg element or CPE/QPE. Birkl et al. [22] shows that it is possible to model diffusion with an RC pair instead of a distributed element in the Randles circuit model. All of these configurations have been exhaustively expounded 

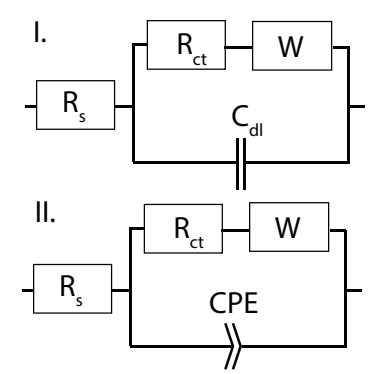
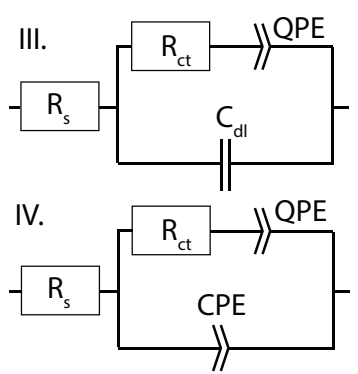

V.

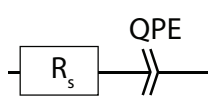

VI.

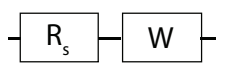

Figure 4: Different configurations of Randles circuits to study regression performance and fitness. $W$ stands for Warburg element. The model of circuits I-IV, relaxation and diffusion are presented together while V-VI only focus on the tail part.

on in [23].

Based on these results, numerous Randles circuits have been evaluated to study the regression performance and fitness. In Fig. 4, the arrangements of circuits I-IV model relaxation and diffusion simultaneously while $\mathrm{V}$ and VI only account for the tail part. The main reason for separation is that the stability and robustness of the impedance regression could be increased using this technique.

The standard Warburg element and CPE/QPE had to be adjusted to match with the non-Fickian diffusion. At first, since only the tail part of the diffusion impedance was modeled, the hyperbolic part of the finite-length Warburg elements was neglected. Hence, the Warburg impedance was simplified to the infinite-length form. Therefore, the Warburg impedance $Z_{\mathrm{w}}$ had to be transformed into a generalized form by replacing the square root in the denominator with $\gamma$. As a result, the generalized Warburg impedance could be written in the following form:

$$
Z_{\mathrm{w}}(\omega)=\frac{R_{\mathrm{w}}}{\left(j \omega \tau_{\mathrm{D}}\right)^{\gamma}}
$$

where $R_{\mathrm{w}}$ denotes the Warburg resistance, $\omega$ stands for the excitation frequency, $\tau_{\mathrm{D}}$ represents the diffusion time constant, $j$ is the imaginary unit and $0 \leq \gamma \leq 1$. Now, the $j^{\gamma}$ term should be practically separated into real and imaginary components to reveal the contribution of $\gamma$ to each part. Using Euler's formula, $Z_{\mathrm{w}}$ was unbundled and grouped into real and imaginary parts:

$$
Z_{\mathrm{w}}(\omega)=\frac{R_{\mathrm{w}}}{\tau_{\mathrm{D}}^{\gamma} \omega^{\gamma}} \cos \left(\frac{\pi}{2} \gamma\right)-\frac{R_{\mathrm{w}}}{\tau_{\mathrm{D}}^{\gamma} \omega^{\gamma}} j \sin \left(\frac{\pi}{2} \gamma\right)
$$

$\tau_{\mathrm{D}}$ used in Eq. 2 was then expressed as

$$
\tau_{\mathrm{D}}=\frac{L_{\mathrm{eff}}^{1 / \gamma}}{D_{\mathrm{eff}}}=\frac{\epsilon_{\mathrm{l}, \mathrm{sep}}^{\beta / \gamma} L_{0}^{1 / \gamma}}{\epsilon_{1, \mathrm{sep}}^{\beta} D_{\mathrm{l}, 0}}
$$

where $L_{\text {eff }}$ denotes the effective diffusion length, $D_{\text {eff }}$ represents the effective diffusion coefficient, $\epsilon_{1, \text { sep }}$ stands for the liquid fraction in the separator, and $\beta$ is the Bruggeman coefficient. Since $\tau_{\mathrm{D}}$ is emphasized from the denominator of Eq. 1, the Warburg coefficient $\sigma$ could be expressed as a fraction of $R_{\mathrm{w}}$ and $\tau_{\mathrm{D}}$ according to

$$
\sigma=\frac{R_{\mathrm{w}}}{\tau_{\mathrm{D}}^{\gamma}}
$$

On the other hand, the transfer functions of CPE and QPE had to be transformed into

$$
\begin{aligned}
& Z_{\mathrm{CPE}}(\omega)=\frac{1}{\left(j \omega \tau_{\mathrm{D}}\right)^{\gamma}} \\
& Z_{\mathrm{QPE}}(\omega)=\frac{1}{\tau_{\mathrm{D}}^{\gamma}(j \omega)^{\gamma}}
\end{aligned}
$$

The updated transfer functions of the distributed elements enabled the non-Fickian diffusion to be properly fitted.

\section{Experimental setup}

This study is devoted to a commercial Samsung ICR $18650-26 \mathrm{~F}$ cell with a nominal capacity of $2600 \mathrm{mAh}$. It consists of a double-sided Nickel-Manganese-Cobalt (NMC) cathode and graphite anode according to the manufacturer's datasheet.

The Samsung ICR 18650-26F cell was evaluated by EIS within the $10 \mathrm{mHz}-100 \mathrm{kHz}$ bandwidth at different States-of-Charge (SoC). The test was run at ambient temperature, namely $25^{\circ} \mathrm{C}$, which was considered to be constant throughout. A Solartron SI1287 (Electrochemical Interface) and a Schlumberger SI 1255 (HF Frequency Response Analyzer) were used for data acquisition. The Nyquist plot of the spectrum and the parametric fitting were produced by ZPlot and ZView software, respectively.

An auxiliary measurement had to be performed along with EIS to determine the cell's Open Circuit Potential (OCP) characteristic at a $0.1 \mathrm{C}$-rated load current. The load current was generated by a TENMA 72-13210 Programmable DC Load in Constant Current (CC) mode. The data was recorded in NI PXI hardware that ran LabVIEW-based data acquisition software.

\section{Analysis of the measurement results}

The purpose of the EIS analysis of the cell was to evaluate the fitness of the different Randles circuit models according to non-Fickian cell impedance data. On the other hand, the cell underwent another EIS measurement to detect how the parameters of the Randles circuit model changed during discharge. The EIS measurements presented in Fig. 5 and 6 were made between $10 \mathrm{mHz}$ and 


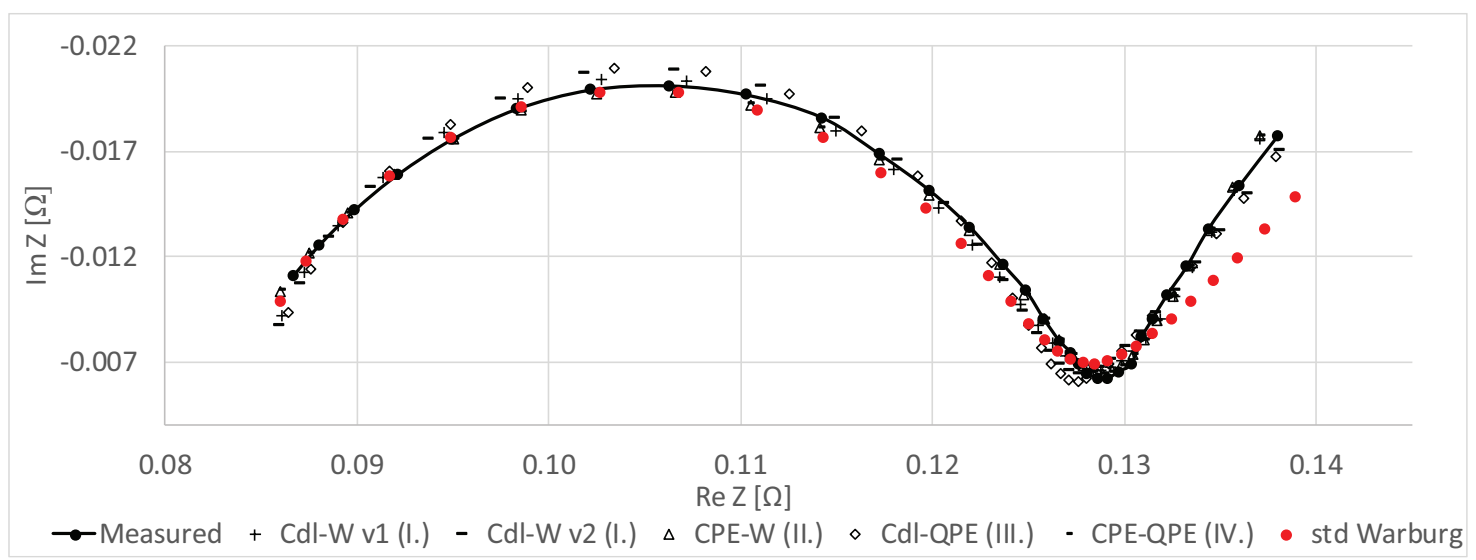

Figure 5: Nyquist plot of the measured impedance and different fit functions (seen in Fig. 4 I-IV). v1 and v2 indicate that Warburg-based models were run on the basis of two different sets of initial parameters. The fit was performed between $13 \mathrm{~Hz}$ and $10 \mathrm{mHz}$ at ambient temperature with a fully charged cell. The "std Warburg" was based on model I in Fig. 4 but with a Warburg exponent of 0.5 .

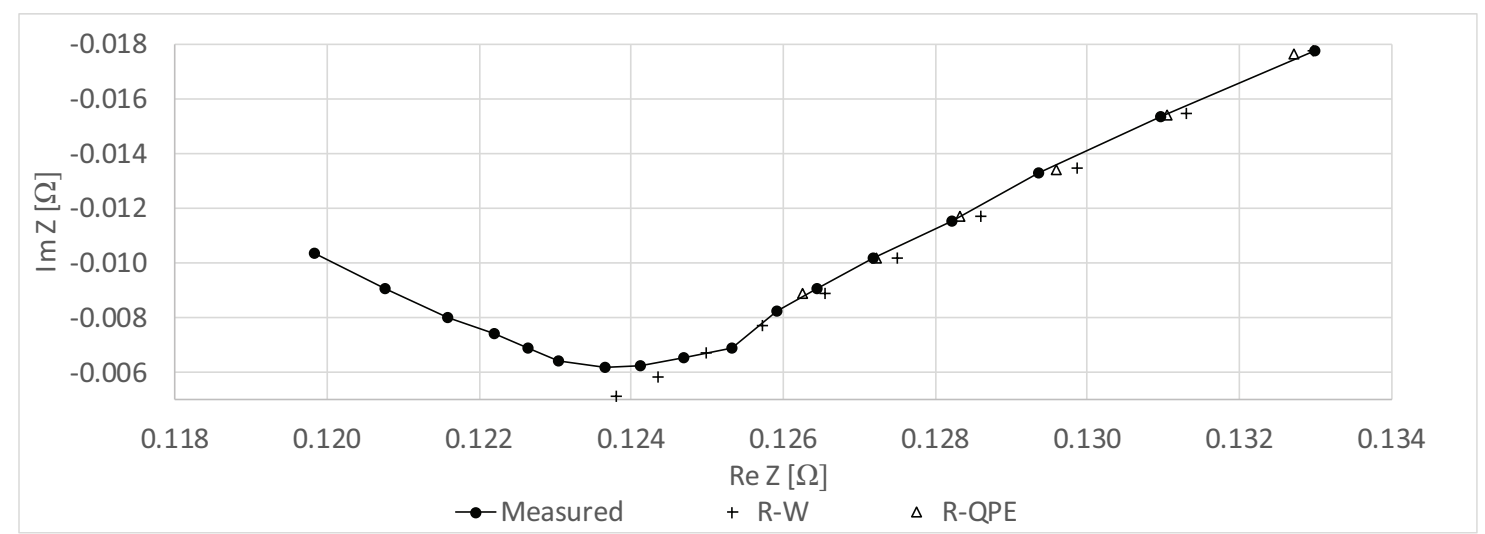

Figure 6: Nyquist plot of the measured impedance and different fit functions (seen in models V-VI of Fig. 4. The fit was performed between $80 \mathrm{mHz}$ and $10 \mathrm{mHz}$ (only the tail part) at ambient temperature with a fully charged cell. The left part of the solid line is associated with the end of the relaxation semicircle and helps to locate the position of the tail.

$13 \mathrm{~Hz}$ where relaxation and diffusion occur. In the case of R-W and R-QPE pairs, only the tail part was modeled. In Fig. 5, only a slight difference between the fits of the model is observed and the CPE-QPE pair shows the best match. Despite the insignificant difference between the non-Fickian models, a substantial improvement in accuracy can be observed especially with regard to fitting the tail when the standard Warburg element was replaced by any of the generalized Warburg- or CPE/QPE-based models.

The changes in the Randles circuit parameters during discharge of the cell was also investigated further where only the best matching CPE-QPE pair was used for fitting. The resultant impedance plot is presented in Fig. 7. The EIS measurements were performed at $20 \%$ SoC level increments. Every step of the discharge was followed by a 12 -hour-long period of relaxation before the EIS measurement was made in order to provide sufficient time for the cell to reach its stationary state. The impedance curves show that the degree of relaxation was high when the rate of the electrode reaction decreased or if the transport of Li ions became limited. This occurred, for example, as the rate of $\mathrm{Li}$ diffusion decreased away from the interface. The evolution of changes in the distinctive impedance curves could be tracked by changes in the parameters of the Randles circuits. Fig. 7 shows that $R_{\mathrm{S}}$ and $R_{\mathrm{ct}}$ significantly increased as SoC decreased due to the decreasing amount of Li-Ion particles engaged in the charge transfer process. Furthermore, the electrolyte resistance $R_{\mathrm{S}}$ increased due to the decreasing ionic conductivity of the solution. All of these results agree with a well-known phenomenon, namely that the overall resistance of the cell increases during discharge. The shape and position of the plateau at the beginning of the semicircle was apparently due to a Solid Electrolyte Interphase (SEI) layer which formed on the anode particles that was unaffected during discharge. The presence of an SEI layer ascertains that the calendar and cycle lives of the cell both reduced. Since the gradient of the tails show significant similarities between $100 \%$ and $20 \%$ SoC, the diffusion time constant and diffusion coefficient of the electrolyte should change slightly during discharge. At $5 \%$ SoC, the 


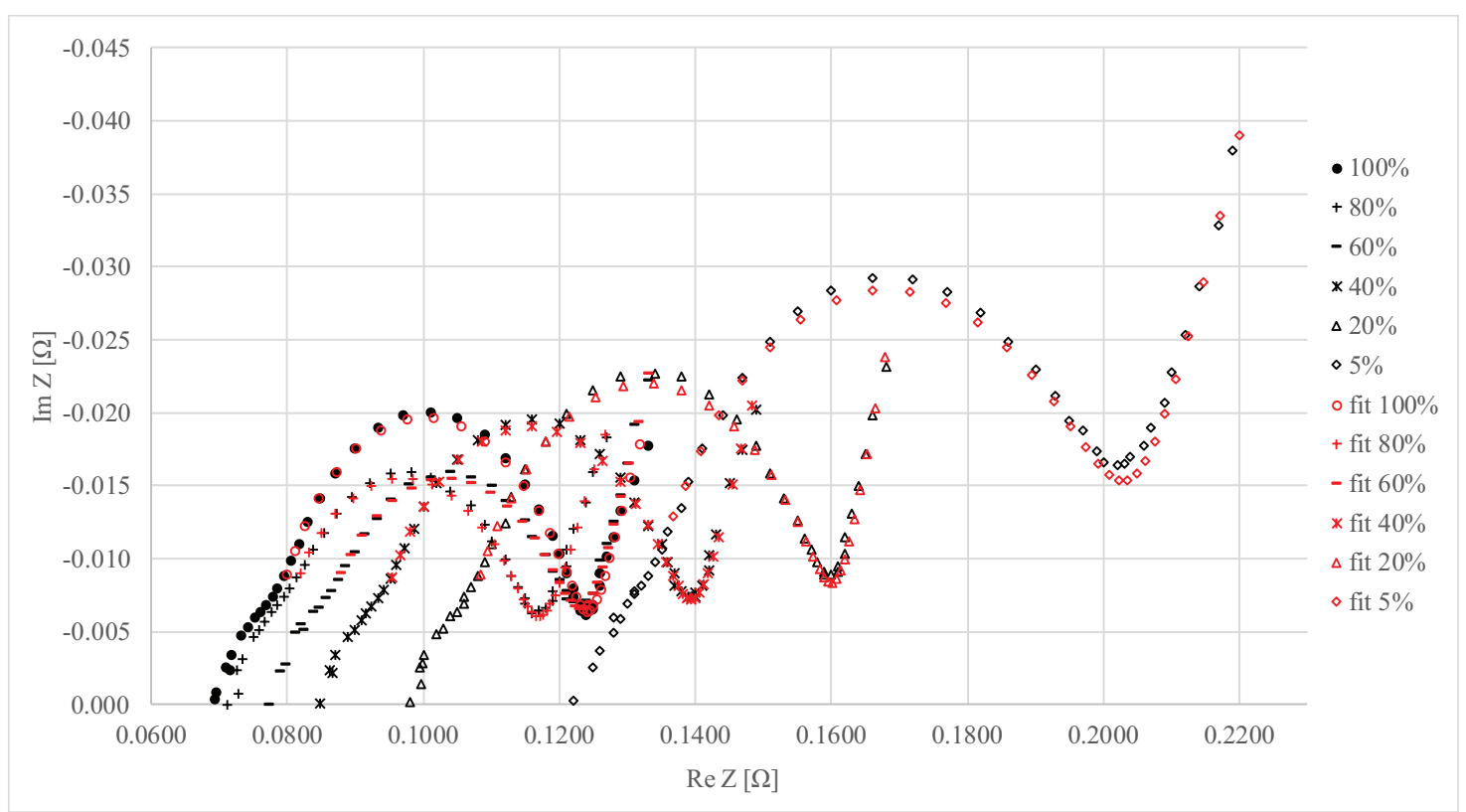

Figure 7: Nyquist plots of the Samsung ICR 18650-26F 2600 mAh Li-Ion cell at different levels of SoC. The temperature was assumed to be constant at $25{ }^{\circ} \mathrm{C}$. The regression bandwidth was limited to between $13 \mathrm{~Hz}$ and $10 \mathrm{mHz}$. The small plateau at approximately $250 \mathrm{~Hz}$ is a consequence of a Solid Electrolyte Interphase (SEI) layer that formed on the anode particles. This shows that the calendar and cycle lives of the cell were reduced. The fit was made by the model of the CPE-QPE pair.

utilizable Li-Ions in the electrode became exhausted leading to a significant decrease in both the rate of diffusion and reaction. On the other hand, the reaction rates did not vary extensively in the normal operating region since the "valley" between the semicircle and tail possesses a similar imaginary component of the impedance.

In order to carry out cell characterization in the time domain, a quasi-equilibrium discharge was run using a 0.1 C-rated load current at a constant temperature of $25{ }^{\circ} \mathrm{C}$. The OCV against SoC curve is presented in Fig. 8 that exhibits typical discharge characteristics with a small plateau around $30 \%$ SoC and rapidly decreases below $10 \%$ SoC.

\subsection{Determining Randles circuit parameters}

Evaluation of EIS data in Fig. 5 and the impedance regression were carried out by ZView software that applies

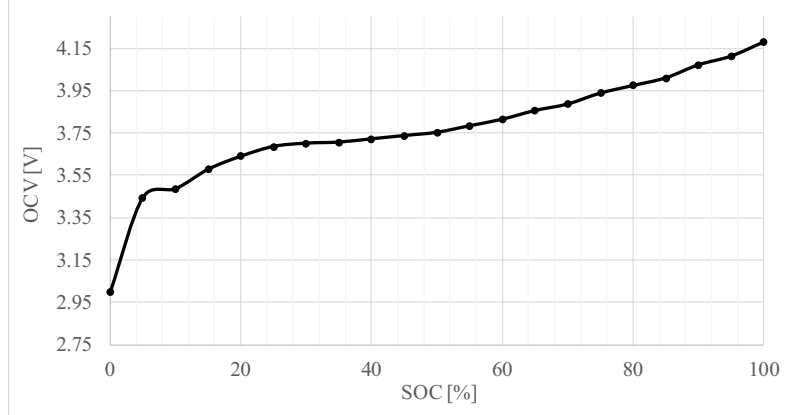

Figure 8: The Open Circuit Voltage (OCV) against Stateof-Charge (SoC) characteristic of the cell. the non-linear least squares method to fit and calculate the Randles circuit parameters. The results summarized in Table 2 show that slight changes in $R_{\mathrm{s}}, R_{\mathrm{ct}}$ and $C_{\mathrm{dl}}$ were observed with this setup. This was due to fitting on an almost ideal semicircle that exhibits a simple RC characteristic in the impedance spectrum. With regard to the estimation of diffusion time constants, diffusion in a real electrochemical battery cell is usually limited due to the relatively thin electrodes. Consequently, from a practical point of view, ZView only has finite-length Warburg elements at its disposal. Since finite-length Warburg elements should require data from the very low bandwidth that is unavailable in the present case, it is favorable to check the applicability of this type of element in the current case.

For this purpose, the Warburg parameters were estimated on the basis of two different sets of initial values as is denoted by v1 and v2 in Fig. 5. Both cases yielded a similar fit but extremely different Warburg parameters. Therefore, the estimation of Warburg parameters ran into multiple local solutions that erroneously characterize the same system with different diffusion time constants. This problem could be efficiently handled by using either the $\mathrm{CPE}+\mathrm{QPE}$ pair or just QPE instead. The results are presented in Table 3. Given the $\gamma_{\mathrm{w}}$ values, the Warburg exponent is clearly far from 0.5 , hence the preliminary assumption of exhibiting non-Fickian diffusion was substantiated. The maximum phase error between the standard Warburg-element- and CPE+QPE pair-based Randles circuit models was $2.3 \%$ at $10 \mathrm{mHz}$. Since Randles circuit parameters are very sensitive due to the origin of their exponential functions, even a small improvement in 
Table 2: The estimated Randles parameters where diffusion was only modeled by a Warburg element and a QPE in two different configurations. The QPE+CPE modeling technique was an effective way to avoid the uncertainties of finite-length Warburg elements due to a lack of data points in the very low bandwidth. The cell was fully charged and kept at $25^{\circ} \mathrm{C}$.

\begin{tabular}{llll}
\hline Randles parameter & QPE+CPE & $W$ & QPE \\
\hline$R_{\mathrm{s}}[\Omega]$ & 0.154 & 0.1968 & 0.1967 \\
$R_{\mathrm{ct}}[\Omega]$ & 0.047 & - & - \\
$C_{\mathrm{dl}}[\mathrm{F}]$ & - & - & - \\
$T_{\mathrm{CPE}}[\mathrm{s}]$ & 2.038 & - & - \\
$\gamma_{\mathrm{CPE}}[-]$ & 0.893 & - & - \\
$\gamma_{\mathrm{QPE}}[-]$ & 0.6868 & - & 0.595 \\
$R_{\mathrm{w}}\left[\Omega \mathrm{m}^{2}\right]$ & - & 0.241 & - \\
$\tau_{\mathrm{w}}[\mathrm{s}]$ & - & 893 & 235.7 \\
$\gamma_{\mathrm{w}}[-]$ & - & 0.595 & - \\
$\tau_{\mathrm{D}}\left[\mathrm{s}^{0.5} / \Omega \mathrm{m}^{2}\right]$ & 339.5 & 893 & 235.7 \\
$\sigma\left[\Omega \mathrm{m}^{2} / \mathrm{s}^{0.5}\right]$ & 0.0015 & 0.00424 & 0.0021 \\
\hline
\end{tabular}

fitting can significantly increase the accuracy in further electrochemical calculations based on these data.

The Randles circuit parameters were measured when the cell was fully charged but changed as the SoC level of the cell was altered as was seen in Fig. 7. The estimated parameters at different SoC levels are summarized in Table 4 and their trends presented in Fig. 9. The changes in $R_{\mathrm{s}}$ and $R_{\mathrm{ct}}$ exhibited an exponential-like decreasing tendency against SoC while $R_{\mathrm{ct}}$ slightly increased at approximately $100 \%$ SoC. The increase in $R_{\mathrm{s}}$ was due to the effect of a reduction in the ionic conductivity, while an increase in $R_{\mathrm{ct}}$ was due to the decreasing rate of LiIon transfer through the electrode-electrolyte interface. In Fig. 10, the characteristics of changes in the CPE capacity and diffusion time constant can be seen. CPE slightly increased with discharge and its overall variance was about $0.4 \mathrm{~F}$. This behavior along with the increase in $R_{\mathrm{ct}}$ can be attributed to the relaxation effect. Furthermore, less charge was available on the anode surface as the cell became fully discharged. The remarkable increase in diffu-

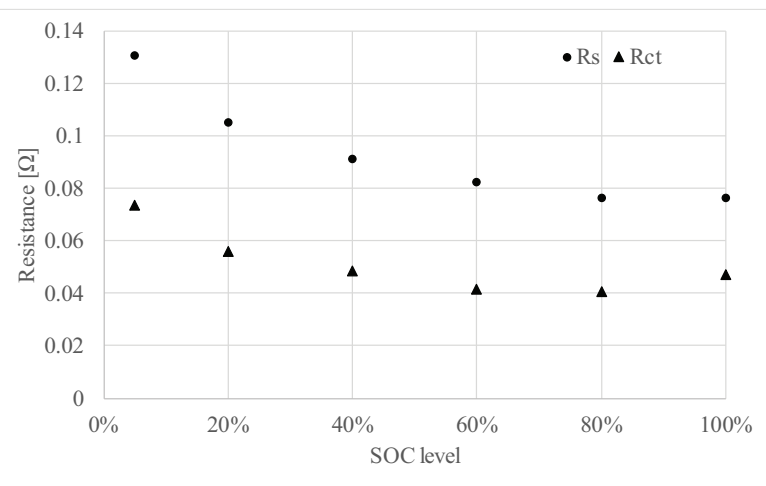

Figure 9: The trend of $R_{\mathrm{S}}$ and $R_{\mathrm{ct}}$ changes with SOC level. The cell shows a well-known increasing overall resistance during discharge.
Table 3: The estimated Randles parameters based on the configurations seen in Fig. 4 where diffusion and doublelayer effect have been modeled by Warburg-element, QPE and CPE in different coupled configurations. In the table heading, $\mathrm{W}$ and $\mathrm{C}$ stand for Warburg and double-layer capacitor, respectively. The cell has been at fully charged state and kept at $25^{\circ} \mathrm{C}$.

\begin{tabular}{lllll}
\hline $\begin{array}{l}\text { Randles } \\
\text { parameter }\end{array}$ & $\mathrm{C}+\mathrm{W}$ & $\mathrm{C}+\mathrm{W}$ & $\mathrm{C}+\mathrm{QPE}$ & $\mathrm{CPE}+\mathrm{W}$ \\
\hline$R_{\mathrm{s}}[\Omega]$ & 0.156 & 0.156 & 0.156 & 0.153 \\
$R_{\mathrm{ct}}[\Omega]$ & 0.041 & 0.041 & 0.041 & 0.047 \\
$C_{\mathrm{d} 1}[\mathrm{~F}]$ & 1.731 & 1.731 & 1.73 & - \\
$T_{\mathrm{CPE}}[\mathrm{s}]$ & - & - & - & 2.038 \\
$\gamma[-]$ & - & - & 0.579 & 0.893 \\
$R_{\mathrm{w}}\left[\Omega \mathrm{m}^{2}\right]$ & 0.0855 & 0.168 & - & 0.263 \\
$\tau_{\mathrm{w}}[\mathrm{s}]$ & 179 & 569.1 & - & 693.2 \\
$\gamma_{\mathrm{w}}[-]$ & 0.578 & 0.5792 & - & 0.687 \\
$\tau_{\mathrm{D}}\left[\mathrm{s}^{0.5} / \Omega \mathrm{m}^{2}\right]$ & 179 & 569.1 & 233.9 & 693.2 \\
$\sigma\left[\Omega \mathrm{m}^{2} / \mathrm{s}^{0.5}\right]$ & 0.0043 & 0.0043 & 0.0023 & 0.0029 \\
\hline & & & & \\
\hline
\end{tabular}

sion time constants at approximately $100 \%$ SoC implies that it was intended that diffusion coefficients should decrease at the end of the discharge. This phenomenon plays a significant role in increasing the overall cell resistance especially when one of the electrodes is exhausted in $\mathrm{Li}$.

\section{Conclusions}

The current work demonstrated an improved method of diffusion modeling. Several configurations of Randles circuits were studied in order to obtain the best fit of the impedance characteristics of a battery. The inappropriate fit of standard Warburg elements with regard to nonFickian diffusion was corrected by applying generalized Warburg elements and CPEs. The proposed generalized model compensates well for the phase error between the measured and modeled impedances.

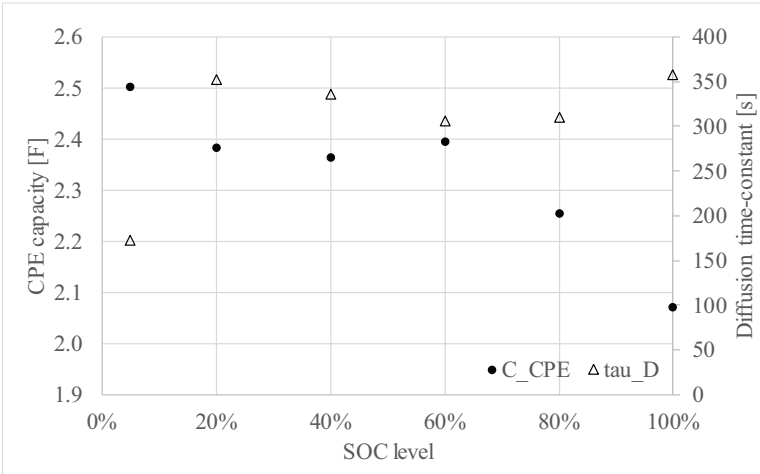

Figure 10: Charactersitics of changes in CPE capacity constants and diffusion time-constant. Diffusion timeconstant remarkably increases during discharge that implies diffusion coefficients to decrease. The CPE does not change significantly. 
Table 4: The estimated Randles circuit parameters at given SoC levels based on the CPE-QPE Randles circuit model.

\begin{tabular}{lllllll}
\hline SoC levels & $100 \%$ & $80 \%$ & $60 \%$ & $40 \%$ & $20 \%$ & $5 \%$ \\
\hline$R_{\mathrm{s}}$ & 0.0764 & 0.0763 & 0.0823 & 0.0909 & 0.105 & 0.1305 \\
$R_{\mathrm{ct}}$ & 0.047 & 0.0406 & 0.0413 & 0.0487 & 0.056 & 0.0734 \\
$C_{\mathrm{CPE}}$ & 2.07 & 2.255 & 2.394 & 2.365 & 2.383 & 2.503 \\
$\tau_{\mathrm{D}}$ & 358 & 310 & 306 & 336 & 353 & 172 \\
\hline
\end{tabular}

The finite-length Warburg element is a classical and generally applied tool in diffusion modeling, but it should be used carefully in some cases. The proposed work also shows that the finite-length Warburg element yields multiple local solutions depending on its initial values if measured impedance data is insufficient within the very low bandwidth of impedance. This leads to ambiguous results in terms of diffusion-related parameters which should be avoided, however, this problem can definitely be solved by applying CPE or QPE instead in diffusion models.

The Randles circuit parameters were estimated and can be used in further calculations to determine the electrochemical parameters of batteries [24].

\section{Symbols}

Binary diffusion coefficient of electrolyte

Warburg coefficient

Diffusion time constant

AC Excitation frequency

Warburg exponent

Electrolyte resistance

Charge transfer resistance

Warburg resistance

Double-layer capacitance

$\begin{array}{ll}D_{\mathrm{l}, 0} & \mathrm{~m}^{2} / \mathrm{s} \\ \sigma & \Omega / \sqrt{\mathrm{s}} \\ \tau_{\mathrm{D}} & \mathrm{s} \\ \omega & \mathrm{Hz} \\ \gamma & - \\ R_{\mathrm{s}} & \Omega \\ R_{\mathrm{ct}} & \Omega \\ R_{\mathrm{w}} & \Omega \\ C_{\mathrm{dl}} & \mathrm{F}\end{array}$

\section{Acknowledgements}

The research was supported by EFOP-3.6.2-16-201700002 "Research of Autonomous Vehicle Systems related to ZalaZone autonome proving ground". We would like to thank Zoltán Lukács and Tamás Kristóf to support conducting EIS measurements in the laboratory of Faculty of Physical Chemistry and sharing their knowledge in electrochemical modeling.

\section{REFERENCES}

[1] Redondo-Iglesias, E.; Venet, P.; Pelissier, S.: Efficiency Degradation Model of Lithium-Ion Batteries for Electric Vehicles, IEEE Transactions on Industry Applications, 2019, 55(2), 1932-1940 DOI: 10.1109/TIA.2018.2877166

[2] Doyle, M.; Fuller, T.F.; Newman, J.: 1-Modeling of Galvanostatic Charge and Discharge, J. Electrochem. Soc., 1993, 140(6), 1526-1533 DOI: 10.1149/1.2221597

[3] Mei, W.; Chen, H.; Sun, J.; Wang, Q.: The effect of electrode design parameters on battery performance and optimization of electrode thickness based on the electrochemical-thermal coupling model, Sustain. Energ. Fuels, 2019, 3(1), 148-165 DOI: 10.1039/c8se00503f

[4] Lawder, M.T.; Northrop, P.W.; Subramanian, V.R.: Model-based SEI layer growth and capacity fade analysis for EV and PHEV batteries and drive cycles, J. Electrochem. Soc., 2014, 161(14), A2099A2108 DOI: 10.1149/2.1161412jes

[5] Orazem, M. E.; Tribollet, B.: Electrochemical Impedance Spectroscopy, John Wiley \& Sons Inc., 2008 ISBN: 9781119363682

[6] Diard, J-P.; Gorrec, L.B.; Montella, C.: Handbook of Electrochemical Impedance Spectroscopy, 2017, 2-40 http: //www.bio-logic.info

[7] Falconi, A.: Electrochemical Li-Ion battery modeling for electric vehicles. Material chemistry. Communaute Universite Grenoble Alpes, 2017, tel01676976

[8] Lasia, A.: Electrochemical Impedance Spectroscopy and its Applications, Springer, 2014 DOI: 10.1007/978-1-4614-8933-7

[9] Barsoukov, E.; Macdonald, J.R.: Impedance Spectroscopy, John Wiley \& Sons, 2005 DOI: 10.1016/j.snb.2007.02.003

[10] Harrington, D.A.: Electrochemical Impedance Spectroscopy (thesis), 2004

[11] Huang, J.: Diffusion impedance of electroactive materials, electrolytic solutions and porous electrodes: Warburg impedance and beyond, Electrochim. Acta, 2018, 281, 170-188 DOI: 10.1016/j.electacta.2018.05.136

[12] Guha, A.; Patra, A.: Online Estimation of the Electrochemical Impedance Spectrum and Remaining Useful Life of Lithium-Ion Batteries, IEEE Transactions on Instrumentation and Measurement, 2018, 67(8), 1836-1849 DOI: 10.1109/TIM.2018.2809138

[13] Huang, J.; Ge, H.; Li, Z.; Zhang, J.: An Agglomerate Model for the Impedance of Secondary Particle in Lithium-Ion Battery Electrode, J. Electrochem. Soc., 2014, 161(8), E3202-E3215 DOI: 10.1149/2.027408jes

[14] Huang, J.; Li, Z.; Zhang, J.; Song, S.; Lou, Z.; $\mathrm{Wu}$, N.: An Analytical Three-Scale Impedance Model for Porous Electrode with Agglomerates in Lithium-Ion Batteries, J. Electrochem. Soc., 2015, 162(4), A585-A595 DOI: 10.1149/2.0241504jes

[15] Franceschetti, D.R.; Macdonald, J.R.: Diffusion of neutral and charged species under small-signal a.c. 
conditions, J. Electroanal. Chem., 1979, 101(3), 307-316 DOI: 10.1016/S0022-0728(79)80042-X

[16] Lelidis, I.; Ross Macdonald, J.; Barbero, G.: Poisson-Nernst-Planck model with Chang-Jaffe, diffusion, and ohmic boundary conditions, J. Phys. D: Appl. Phys., 2016, 49(2), 25503 DOI: 10.1088/00223727/49/2/025503

[17] Sapoval, B.; Chazalviel, J.-N.; Peyrière, J.: Electrical response of fractal and porous interfaces, Phys. Rev. A, 1988, 38(11), 5867-5887 DOI: 10.1103/PhysRevA.38.5867

[18] Jacobsen, T.; West, K.: Diffusion Impedance in Planar, Cylindrical and Spherical Symmetry, Electrochim. Acta, 1995, 40(2), 255-262 DOI: 10.1016/0013-4686(94)E0192-3

[19] Bisquert, J.; Garcia-Belmonte, G.; Bueno, P.; Longo, E.; Bulhões, L.O.: Impedance of constant phase element (CPE)-blocked diffusion in film electrodes, J. Electroanal. Chem., 1998, 452(2), 229234 DOI: 10.1016/S0022-0728(98)00115-6

[20] Ramos-Barrado, J.R.; Galán Montenegro, P.; Cri- ado Cambón, C.: A generalized Warburg impedance for a nonvanishing relaxation process, J. Chem. Phys., 1996, 105(7), 2813-2815 DOI: 10.1063/1.472806

[21] Qu, D.: The study of the proton diffusion process in the porous $\mathrm{MnO} 2$ electrode, Electrochim. Acta, 2004, 49(4), 657-665 DOI: 10.1016/j.electacta.2003.08.030

[22] Birkl, C.R.; Howey, D.A.: Model identification and parameter estimation for $\mathrm{LiFePO} 4$ batteries, IET Conference Publications, 2013, 2013(621 CP), 1-6 DOI: $10.1049 /$ cp.2013.1889

[23] Zou, C.; Zhang, L.; Hu, X.; Wang, Z.; Wik, T.; Pecht, M.: A review of fractional-order techniques applied to lithium-ion batteries, lead-acid batteries, and supercapacitors, J. Power Sources, 2018, 390(June), 286-296 DOI: 10.1016/j.jpowsour.2018.04.033

[24] Nguyen, T.Q.; Breitkopf, C.: Determination of diffusion coefficients using impedance spectroscopy data, J. Electrochem. Soc., 2018, 165(14), E826E831 DOI: $10.1149 / 2.1151814$ jes 